\title{
The contact angle of the colloidal liquid-gas interface and a hard wall
}

\author{
Paul P F Wessels ${ }^{1}$, Matthias Schmidt ${ }^{2,3}$ and Hartmut Löwen ${ }^{1}$ \\ ${ }^{1}$ Institut für Theoretische Physik II, Heinrich-Heine-Universität Düsseldorf, Universitätsstraße 1, \\ 40225 Düsseldorf, Germany \\ 2 Debye Institute, Soft Condensed Matter Group, Utrecht University, Princetonplein 5, \\ 3584 CC Utrecht, The Netherlands \\ E-mail: wessels@thphy.uni-duesseldorf.de
}

Received 6 May 2004

Published 10 September 2004

Online at stacks.iop.org/JPhysCM/16/S4169

doi:10.1088/0953-8984/16/38/030

\begin{abstract}
We consider the Asakura-Oosawa-Vrij model of hard sphere colloids and ideal polymer coils in contact with a planar hard wall at (colloidal) liquid-gas coexistence. Using extensive numerical density functional calculations, the liquid-gas, wall-liquid and wall-gas interfacial free energies are calculated. The results are inserted into Young's equation to obtain the contact angle between the liquid-gas interface and the wall. As a function of polymer fugacity this angle exhibits discontinuities of slope ('kinks') upon crossing first-order surface phase transitions located on the gas branch of the bulk binodal. Each kink corresponds to a transition from $n-1$ to $n$ colloid layers adsorbed at the wall, referred to as the $n$th layering transition. The corresponding adsorption spinodal points from $n-1$ to $n$ layers upon reducing the polymer fugacity along the bulk binodal were found in a previous study (Brader et al 2002 J. Phys.: Condens. Matter. 14 L1; Brader et al 2003 Mol. Phys. 101 3349). Remarkably, we find desorption spinodal points from $n$ to $n-1$ layers to be absent upon increasing polymer fugacity at bulk coexistence, and many branches (containing up to seven colloid layers) remain metastable. Results for the first layering binodal and both spinodal branches off bulk coexistence hint at a topology of the surface phase diagram consistent with these findings. Both the order of the transition to complete wetting and whether it is preceded by a finite or an infinite number of layering transitions remain open questions. We compare the locations of the first layering binodal line and of the second layering binodal point at bulk coexistence with recent computer simulation results by Dijkstra and van Roij (2002 Phys. Rev. Lett. 89 208303) and discuss our results for the contact angle in the light of recent experiments.
\end{abstract}

3 On leave from: Institut für Theoretische Physik II, Heinrich-Heine-Universität Düsseldorf, Universitätsstraße 1, 40225 Düsseldorf, Germany. 


\section{Introduction}

In suspensions of sterically stabilized colloidal particles mixed with non-adsorbing globular polymers, the latter induce an effective attraction between the colloids due to the depletion effect [1]. Such mixtures can phase separate into two fluid phases, one being a colloidal liquid that is rich in colloids and poor in polymers and the other being a colloidal gas that is poor in colloids and rich in polymers. Colloid-polymer mixtures serve as excellent model systems in which to study many phenomena associated with liquid-gas phase separation as the scales of time and length are much larger than in atomic and molecular systems [1,2]. Recent experiments have focused on the bulk phase behaviour (see [1] and references therein), (colloidal) liquid-gas interface tension [3-7], capillary wave fluctuations observed in real space [8], droplet coalescence [8] and further non-equilibrium phenomena $[1,7,9]$. The behaviour at non-adsorbing walls has been studied by measuring the contact angle between the free liquid-gas interface and a substrate acting as a hard wall. Complete wetting of the wall by the colloidal liquid has been observed for a wide variety of state points [9]. However, there are also reports of a transition from partial to complete wetting $[10,11]$; hence this remains an interesting topic.

Most of the essential physics of colloid-polymer mixtures is captured by the AsakuraOosawa-Vrij (AO) model of hard sphere colloids and ideal polymers [12-14], which has become a widely used reference system. Theoretical approaches [15, 16] and computer simulations [17-23] have given insight into its bulk phase behaviour, and some recent work [20, 24] aims at including more realistic colloid-polymer and polymer-polymer interactions. Studies based on a one-component description of colloids interacting with an effective depletion potential (obtained by integrating out the polymer degrees of freedom and truncating at the pairwise level) were devoted to inhomogeneous situations, such as the free fluid-fluid interface [25, 26] and adsorption at a hard wall [27]. Following the development of an accurate density functional theory (DFT) specific to the binary AO model $[28,29]$, further research was stimulated in inhomogeneous situations such as liquid-gas $[30,31]$ and wallfluid interfaces [30-32] and results were compared to those from simulations [21-23, 33]. In particular, in $[30,31]$ the AO model was considered for contact with a planar hard wall. A sequence of first-order layering transitions was found on the gas branch of the (liquid-gas) binodal upon reducing the polymer fugacity. Further reducing the polymer fugacity leads to a transition to complete wetting of the wall by colloidal liquid. This scenario was corroborated by a simulation study [21]. The relation of the results from these different approaches will be reexamined in the light of the findings of the present study in more detail below. The adsorption properties at a wall are intimately related to the wall-fluid interfacial free energies (or 'wall tensions'), for which an analytical expression was obtained from a scaled-particle treatment and which was found to compare well with results from full numerical DFT calculations [32]. However, despite its experimental accessibility [7, 9-11], the contact angle of the free liquidgas interface and a hard planar wall has not been considered either via theory or simulations, in contrast to wetting behaviour $[21,30,31,34]$. The aim of the present study is to obtain a quantitative understanding of the contact angle and elucidate its relation to the surface phase behaviour on the basis of the AO model.

We obtain the (macroscopic) contact angle, $\theta$, from the liquid-gas (lg), the wall-gas (wg) and the wall-liquid (wl) interface tensions, $\gamma_{\mathrm{lg}}, \gamma_{\mathrm{wg}}$ and $\gamma_{\mathrm{wl}}$, respectively, via Young's equation [35],

$$
\cos \theta=\frac{\gamma_{\mathrm{wg}}-\gamma_{\mathrm{wl}}}{\gamma_{\mathrm{lg}}}
$$

A depletion attraction similar to that acting between two colloids acts between one colloid and a hard wall [27]; the latter therefore favours the colloidal liquid. As a consequence, we expect 
$\gamma_{\mathrm{wg}}>\gamma_{\mathrm{wl}}$ everywhere at coexistence, and $\theta<\pi / 2$. For state points where $\gamma_{\mathrm{wg}}<\gamma_{\mathrm{wl}}+\gamma_{\mathrm{lg}}$, the contact angle $\theta>0$ and the surface is partially wetted by the liquid. However, as soon as $\gamma_{\mathrm{wg}}=\gamma_{\mathrm{wl}}+\gamma_{\mathrm{lg}}$ a macroscopic liquid layer will intrude between the gas and the wall and the latter is completely wetted by the liquid. The transition from partial to complete wetting induced by changing an appropriate thermodynamic variable is referred to as the wetting transition [36, 37]. A study of $\theta$ can supply a link between theoretical predictions of surface phase behaviour and experiments and we display our central result in figure 6 .

The paper is organized as follows. In section 2 we define the model and discuss in section 3 the density functional theory used to calculate the interface tensions. In section 4 we present results and we conclude in section 5 .

\section{Model}

We consider the Asakura-Oosawa-Vrij (AO) model of $N_{\mathrm{c}}$ hard sphere colloids and $N_{\mathrm{p}}$ ideal polymers in a volume $V$. The colloids (species c) and polymers (species p) have diameters $\sigma_{i}$, bulk packing fractions $\eta_{i}=N_{i} V_{i} / V$ and particle volumes $V_{i}=(\pi / 6) \sigma_{i}^{3}$ for $i=\mathrm{c}, \mathrm{p}$, respectively. The colloid-colloid as well as the colloid-polymer interaction potentials are those of hard spheres, so $u_{i j}(r)=\infty$ when $r<\left(\sigma_{i}+\sigma_{j}\right) / 2$ and $u_{i j}(r)=0$ otherwise, with $i j=\mathrm{cc}, \mathrm{cp}$. The polymers do not interact with each other, i.e. $u_{\mathrm{pp}}(r)=0$ for all $r$. Due to the non-additive ranges of these interaction potentials, the polymers induce an effective attraction between the colloids, which for sufficiently large size ratios $\sigma_{\mathrm{p}} / \sigma_{\mathrm{c}}(\gtrsim 0.35)$ drives a thermodynamically stable phase separation into a colloid-rich (liquid) and a colloid-poor (gas) phase $[15,16]$. All bare interactions are of an entropic nature, and therefore the temperature $T$ does not play a role. The only relevant model parameter is the size ratio $q=\sigma_{\mathrm{p}} / \sigma_{\mathrm{c}}$. We often use the so-called polymer reservoir representation where the mixture is in contact with a polymer reservoir, which determines the polymer chemical potential. In this situation, the thermodynamic state parameters are $\eta_{\mathrm{c}}$ and $\eta_{\mathrm{p}}^{\mathrm{r}}$, the latter being the polymer packing fraction in the reservoir which is proportional to the polymer fugacity as these particles are ideal.

\section{Density functional theory}

We use the fundamental measure density functional for the AO model $[28,29]$ to calculate colloid and polymer density profiles from which the interface tensions can then be obtained. In density functional theory (DFT), the grand-canonical free energy is expressed as a functional, $\Omega\left[\rho_{\mathrm{c}}(\mathbf{r}), \rho_{\mathrm{p}}(\mathbf{r})\right]$, of the one-particle distribution functions $\rho_{i}(\mathbf{r})$ (with $\left.i=\mathrm{c}, \mathrm{p}\right)$, given by [38]

$$
\begin{aligned}
\Omega\left[\rho_{\mathrm{c}}(\mathbf{r}), \rho_{\mathrm{p}}(\mathbf{r})\right] & =F_{\mathrm{exc}}\left[\rho_{\mathrm{c}}(\mathbf{r}), \rho_{\mathrm{p}}(\mathbf{r})\right]+k_{\mathrm{B}} T \sum_{i=\mathrm{c}, \mathrm{p}} \int \mathrm{d} \mathbf{r} \rho_{i}(\mathbf{r})\left[\ln \left(\rho_{i}(\mathbf{r}) \Delta_{i}\right)-1\right] \\
+ & \sum_{i=\mathrm{c}, \mathrm{p}} \int \mathrm{d} \mathbf{r} \rho_{i}(\mathbf{r})\left[u_{\mathrm{ext}, i}(\mathbf{r})-\mu_{i}\right],
\end{aligned}
$$

where $k_{\mathrm{B}}$ is Boltzmann's constant, $\Delta_{i}$ is the 'thermal volume' of species $i$, i.e. the third power of the de Broglie wavelength, and $u_{\text {ext }, i}(\mathbf{r})$ and $\mu_{i}$ are the external potential and the chemical potential for species $i$, respectively. The excess Helmholtz free energy functional, $F_{\text {exc }}=\int \mathrm{d} \mathbf{r} \Phi(\mathbf{r})$, with $\Phi$ the excess free energy density, is given in $[28,29]$ and is not reproduced here ${ }^{4}$.

4 We do not include the tensorial weight function in the present calculations. 
In thermodynamic equilibrium, the functional is stationary, $\delta \Omega / \delta \rho_{i}(\mathbf{r})=0$ (with $i=\mathrm{c}, \mathrm{p}$ ), and the resulting equations yield the stable distributions, i.e.

$$
\rho_{i}(\mathbf{r})=z_{i} \exp \left[-\beta u_{\mathrm{ext}, i}(\mathbf{r})-\beta \frac{\delta F_{\mathrm{exc}}\left[\left\{\rho_{j}(\mathbf{r})\right\}\right]}{\delta \rho_{i}(\mathbf{r})}\right],
$$

with $z_{i}=\Delta_{i}^{-1} \exp \left[\beta \mu_{i}\right]$ the fugacity of component $i$ and $\beta=1 / k_{\mathrm{B}} T$. The equilibrium distribution functions are normalized, $\int \mathrm{d} \mathbf{r} \rho_{i}(\mathbf{r})=N_{i}$. We note that the polymer fugacity is proportional to the polymer packing fraction in the polymer reservoir, $\eta_{\mathrm{p}}^{\mathrm{r}}=z_{\mathrm{p}} V_{\mathrm{p}}$, and we usually refer to $\eta_{\mathrm{p}}^{\mathrm{r}}$ as the polymer fugacity. We have solved these equations numerically for $\rho_{i}(z)$ in one spatial dimension $z$ for the free liquid-gas interface (where $u_{\text {ext }, i}(z)=0$ everywhere) as well as both for the liquid and the gas at bulk coexistence in the presence of the external hard wall potential, i.e. for $u_{\mathrm{ext}, i}(z)=\infty$ for $z<\sigma_{i} / 2$ and $u_{\mathrm{ext}, i}(z)=0$ otherwise, for both species $i=\mathrm{c}, \mathrm{p}$, where $z$ is the space coordinate perpendicular to the wall. The numerical routine we have used is a Picard iteration procedure with a Broyles mixing scheme [39]. The 'mixing parameter' is continuously adapted to obtain optimal convergence. Additionally, it is important to realize that in situations with several metastable minima, as we find to occur for the coexisting gas in contact with the hard wall, the initial guess for the profiles in the iteration procedure determines to which minimum the routine converges.

Once the density profiles are known, the interface tension is given by $\gamma=\left(\Omega_{\mathrm{inh}}+P V\right) / A$, where $\Omega_{\text {inh }}=\Omega\left[\rho_{\mathrm{c}}(\mathbf{r}), \rho_{\mathrm{p}}(\mathbf{r})\right]$ (i.e. the functional, equation (2), evaluated at the solutions of equation (3)) is the grand-canonical free energy of the inhomogeneous system, $P$ is the bulk pressure and $A$ is the lateral (perpendicular to the $z$-direction) system area. In terms of density profiles this quantity can be written as

$$
\gamma=\int \mathrm{d} z[\omega(z)+P]
$$

where

$$
\omega(z)=k_{\mathrm{B}} T \sum_{i=\mathrm{c}, \mathrm{p}} \rho_{i}(z)\left[\ln \left(\rho_{i}(z) \Delta_{i}\right)-1\right]-\sum_{i=\mathrm{c}, \mathrm{p}} \mu_{i} \rho_{i}(z)+k_{\mathrm{B}} T \Phi(z)
$$

can be viewed as a local grand potential density (evaluated with the minimized density profiles). In case of the liquid-gas interface, the integral in equation (4) is over all space, i.e. $z$ runs from $-\infty$ (bulk gas) to $\infty$ (bulk liquid). In the case of the fluid in contact with a hard wall, the integral runs from $z=0$ (at the actual location of the hard wall) to $\infty$ (bulk). In the numerical routine, we compute the interface tensions for each state point for different system sizes and refined termination criteria for the iteration. This gives an estimate of the numerical error in the result for the interface tensions, which is important as the resulting contact angle can be very sensitive to these errors. Often the dividing surface [35] is chosen at $z=\sigma_{\mathrm{c}} / 2$; then subtraction of $\sum_{i=\mathrm{c}, \mathrm{p}} P \sigma_{i}$ from the present definition of the wall-fluid tension, equation (4), is required. For our present goal this is irrelevant as this term does not affect $\theta$, as it drops out of the numerator in equation (1).

\section{Results}

\subsection{Layered states at the hard wall}

We have calculated colloid and polymer density profiles of the free liquid-gas interface, as well as the wall-gas and the wall-liquid interfacial profiles at bulk coexistence. For a given state point the liquid-gas and the wall-liquid profiles are both unique (disregarding trivial translations of the liquid-gas interface); such results have been presented elsewhere [30, 31]. 


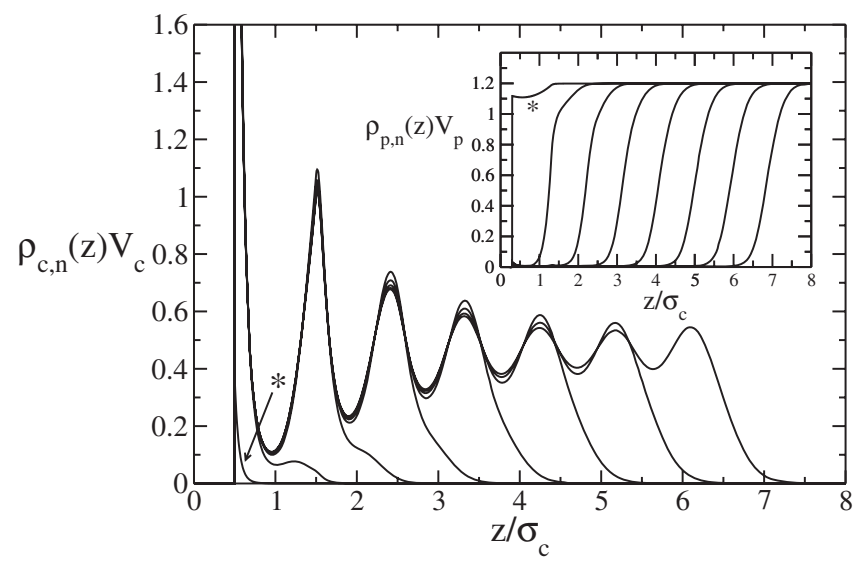

Figure 1. Colloid density profiles, $\rho_{\mathrm{c}, n}(z) V_{\mathrm{c}}$, at a hard wall as a function of the scaled distance, $z / \sigma_{\mathrm{c}}$, from the wall at the gas branch of the liquid-gas binodal for $q=0.6$ and $\eta_{\mathrm{p}}^{\mathrm{r}}=1.2$. Shown are results for $n$-layer states with $n=0,1,2, \ldots, 7$ (left to right). All states are metastable except for the globally stable $n=0$-layer state (see the small peak at contact with the wall, marked with an asterisk). The inset shows the corresponding polymer profiles (also from left to right, with an asterisk the marking $n=0$-layer state), $\rho_{\mathrm{p}, n}(z) V_{\mathrm{p}}$, as a function of $z / \sigma_{\mathrm{c}}$. The normalizations of the density profiles are such that in bulk they reduce to the packing fractions, $\lim _{z \rightarrow \infty} \rho_{i, n}(z) V_{i}=\eta_{i}$.

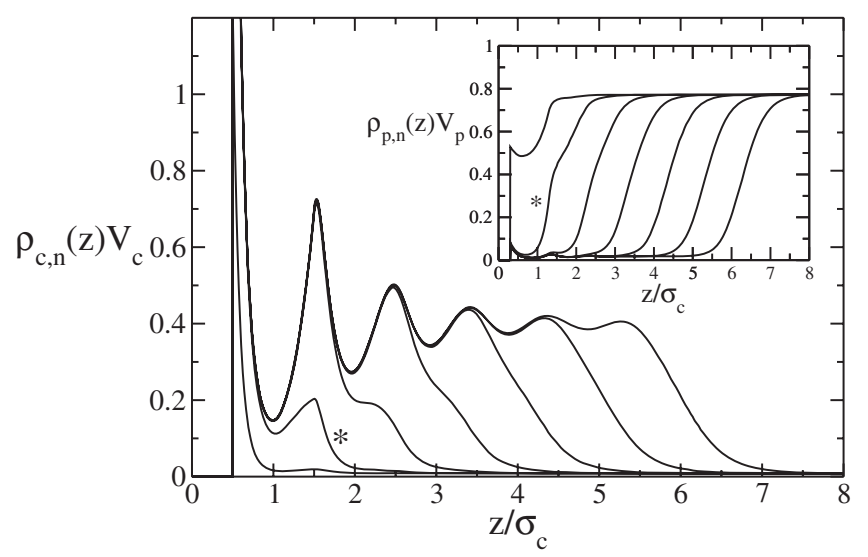

Figure 2. The same as figure 1 , but for $\eta_{\mathrm{p}}^{\mathrm{r}}=0.8$ and $n=0,1,2, \ldots, 6$ (left to right). All profiles are metastable except for the globally stable $n=1$ state (marked with asterisks).

However, in the case of the coexisting gas in contact with the hard wall, we have found many metastable states, each corresponding to an integer number of layers, $n$, of colloids adsorbed at the wall. In figures 1 and 2, we have plotted a number of such profiles, denoted by $\rho_{\mathrm{c}, n}(z)$, for size ratio $q=0.6$ and fugacities $\eta_{\mathrm{p}}^{\mathrm{r}}=1.2$ and 0.8 , respectively, along with the corresponding polymer profiles, $\rho_{\mathrm{p}, n}(z)$, given in the respective insets (for reference, the bulk phase diagram for $q=0.6$ is given in the inset of figure 9). For $\eta_{\mathrm{p}}^{\mathrm{r}}=1.2$ the zero-layer state (marked with an asterisk in figure 1) exhibits practically no excess colloid adsorption and is the globally stable state. The grand potential, and hence the interface tension, increases with the number of layers, i.e. $\gamma_{\mathrm{wg}, 0}<\gamma_{\mathrm{wg}, 1}<\gamma_{\mathrm{wg}, 2}<\cdots<\gamma_{\mathrm{wg}, 7}$, where $\gamma_{\mathrm{wg}, n}$ is the wall-gas tension corresponding to $n$ colloid layers (as given in equation (4) and evaluated with $\rho_{i, n}(z), i=\mathrm{c}, \mathrm{p}$ ). 


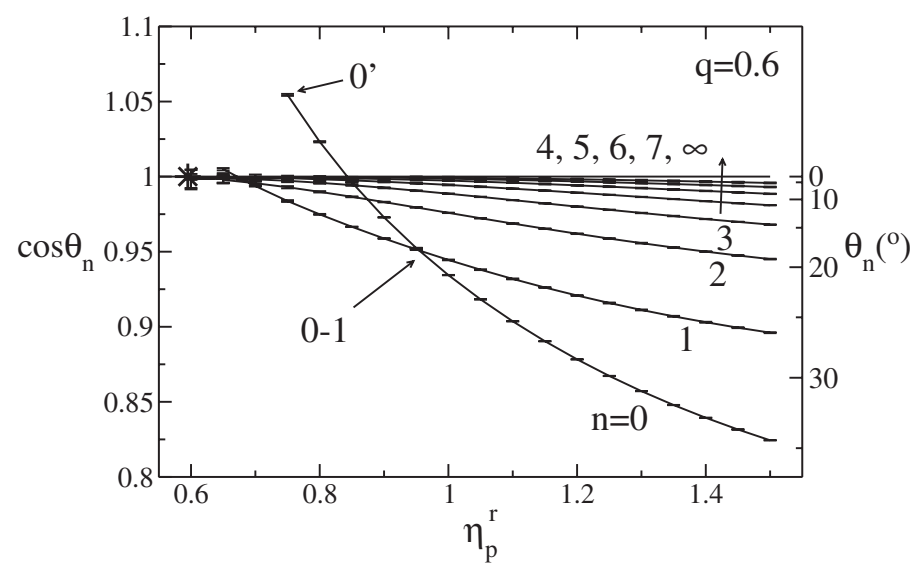

Figure 3. Cosines of the contact angle, $\cos \theta_{n}$ as defined via Young's equation as $\left(\gamma_{\mathrm{wg}}-\gamma_{\mathrm{wl}}\right) / \gamma_{\mathrm{lg}}$, as a function of the polymer reservoir packing fraction, $\eta_{\mathrm{p}}^{\mathrm{r}}$, for size ratio $q=0.6$. A scale of the bare angle (in degrees, ${ }^{\circ}$ ) is given on the right vertical axis. Shown are branches, $\theta_{n}$, corresponding to $n$ colloid layers adsorbed at the wall-gas interface at liquid-gas coexistence (with $n=0,1,2, \ldots, 7$, increasing in the direction of the arrow). The constant $\cos \theta_{n}=1\left(\theta_{n}=0^{\circ}\right)$ is marked $\infty$ and corresponds to two macroscopically separated wall-liquid and liquid-gas interfaces. For every value of $\eta_{\mathrm{p}}^{\mathrm{r}}$, the branch corresponding to the lowest $\cos \theta_{n}$ (i.e. the largest contact angle $\theta_{n}$ ) yields the thermodynamically stable state; all other branches are metastable. The crossing point of $\theta_{0}$ and $\theta_{1}$ (marked 0-1) denotes the first layering transition and the spinodal point of the zero-layer branch is marked $0^{\prime}$. Also indicated is the location of the wetting transition (star) according to Brader et al $[30,31]$.

For $\eta_{\mathrm{p}}^{\mathrm{r}}=0.8$ the equilibrium profile is given by $\rho_{i, 1}(z)$ (marked with an asterisk in figure 2). Remarkably, in this case, the solution $\rho_{i, 0}(z)$ corresponds to a higher tension than all others, i.e. $\gamma_{\mathrm{wg}, 1}<\gamma_{\mathrm{wg}, 2}<\cdots<\gamma_{\mathrm{wg}, 6}<\gamma_{\mathrm{wg}, 0}$. The two state points considered $\left(\eta_{\mathrm{p}}^{\mathrm{r}}=0.8,1.2\right)$ are at polymer fugacities larger than that at which the Fisher-Widom line [40, 41] hits the bulk binodal (at $\eta_{\mathrm{p}}^{\mathrm{r}} \approx 0.533$ for $q=0.6$ [29]), which implies that correlations decay asymptotically in an oscillatory fashion in the liquid phase. Apparently, this oscillatory nature also appears in the effective interface potential between the wall and the liquid-gas interface, yielding many metastable minima [31], see [42].

\subsection{The wall contact angle of the liquid-gas interface}

We have calculated the liquid-gas interface tension, $\gamma_{l g}$, the wall-liquid tension, $\gamma_{\mathrm{wl}}$, and the wall-gas tensions for all $n$-layer states identified, $\gamma_{\mathrm{wg}, n}$, for the full range of polymer fugacities at bulk liquid-gas coexistence. The results have been inserted into Young's equation, equation (1), yielding a contact angle curve, $\theta_{n}$, for each $n$-layer state. The results for $q=0.6$ are plotted in figure 3 and a magnification of the region close to $\cos \theta_{n}=1$ is displayed in figure 4. From the definition of the contact angle, equation (1), and the fact that $\gamma_{\mathrm{lg}}$ and $\gamma_{\mathrm{wl}}$ are unique for each state point, $\eta_{\mathrm{p}}^{\mathrm{r}}$, the state $n$ with the lowest free energy also possesses the lowest value of $\cos \theta_{n}$. Hence, for high $\eta_{\mathrm{p}}^{\mathrm{r}}$ the equilibrium contact angle is given by $\theta_{0}$ (corresponding to the zero-layer state); see figure 3 . Decreasing $\eta_{\mathrm{p}}^{\mathrm{r}}$, leads to an increase in $\cos \theta_{0}$, until it crosses the $\cos \theta_{1}$ branch and becomes metastable. The crossing point, where $\theta_{0}=\theta_{1}$, denotes the $0-1$ layering transition and is also referred to as the first layering transition. (Consistent with [30,31], the surface phase transition from $n-1$ to $n$ adsorbed colloid layers at the wall-gas interface is referred to as the $n$th layering transition.) Upon further decreasing $\eta_{\mathrm{p}}^{\mathrm{r}}$ (see figure 4), 


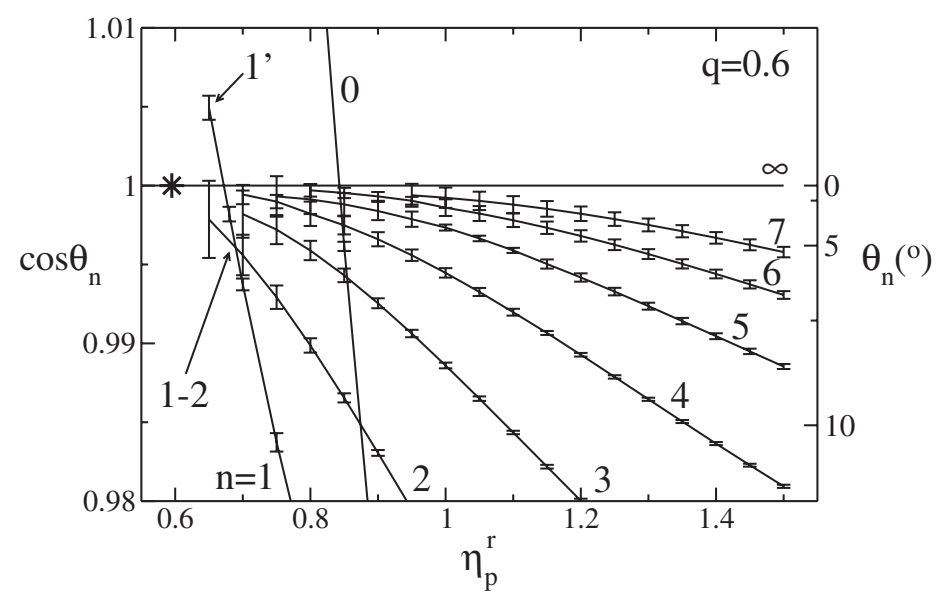

Figure 4. As figure 3, but magnified close to $\cos \theta_{n}=1$. For the sake of clarity, some of the points of the three-, four- and five-layer branches close to the wetting transition are omitted. Indicated is the location of the second layering transition (marked 1-2) and the one-layer spinodal point (1').

$\cos \theta_{1}$ is in turn crossed by $\cos \theta_{2}$ and the crossing point, where $\theta_{1}=\theta_{2}$, is the $1-2$ (second) layering transition. This scheme suggests that there could well be further layering transitions upon reducing $\eta_{\mathrm{p}}^{\mathrm{r}}$, and this impression is strengthened by the fact that the (metastable) states $\cos \theta_{n}$ (with $n$ from 3 to 7; see figure 4) all seem to converge to around the location of the wetting transition. However, we have not been able to resolve the third $[30,31]$ and (possible) higher layering transitions and we note that any of these should be located in the small region of $\cos \theta$ between 0.995 and 1 with $\eta_{\mathrm{p}}^{\mathrm{r}}$ between 0.6 and 0.65 ; see figure 4 . Consequently, we can also not obtain insight into the nature of the transition to complete wetting, i.e. whether this is second order and occurs via an infinite sequence of layering transitions or is first order and is preceded by only a finite number of layering transitions. For a more extensive discussion of these two possible scenarios, we refer the reader to [31].

Upon reducing $\eta_{\mathrm{p}}^{\mathrm{r}}$, metastable zero-layer states can be tracked into a region where the contact angle takes unphysical values, $\cos \theta_{0}>1$ (figure 3). The same happens for onelayer states as can be seen in figure 4. Inserting equilibrium values of the interface tensions obtained from DFT into equation (1) ensures that $\cos \theta \leqslant 1$, but this does not need to be the case when using interface tensions of metastable states. Reducing $\eta_{\mathrm{p}}^{\mathrm{r}}$ even further, the metastable zero-layer state eventually becomes unstable at an adsorption spinodal point; see figure 3. Beyond this point (for even lower $\eta_{\mathrm{p}}^{\mathrm{r}}$ ), no zero-layer state can be stabilized and the numerical iteration rather converges to the one-layer state. Similar adsorption spinodal points were found for higher $n$ and we have located those with a (moderate) resolution of 0.05 in $\eta_{\mathrm{p}}^{\mathrm{r}}$. From the present data the adsorption spinodal fugacities for $n$-layer states, $\eta_{\mathrm{p}, n^{\prime}}^{\mathrm{r}}$, are $\eta_{\mathrm{p}, 0^{\prime}}^{\mathrm{r}}=0.75, \eta_{\mathrm{p}, 1^{\prime}}^{\mathrm{r}}=\eta_{\mathrm{p}, 2^{\prime}}^{\mathrm{r}}=0.65$ and $\eta_{\mathrm{p}, 3^{\prime}}^{\mathrm{r}}=\eta_{\mathrm{p}, 4^{\prime}}^{\mathrm{r}}=\eta_{\mathrm{p}, 5^{\prime}}^{\mathrm{r}}=0.6$. For states with an even thicker colloid film ( $n=6$ and 7), the profiles no longer converged properly at low $\eta_{\mathrm{p}}^{\mathrm{r}}$, and we have not been able to obtain precise values for $\eta_{\mathrm{p}, 6^{\prime}}^{\mathrm{r}}$ and $\eta_{\mathrm{p}, 7^{\prime}}^{\mathrm{r}}$. The evolution for surface states upon increasing $\eta_{\mathrm{p}}^{\mathrm{r}}$ is in striking contrast. No spinodal points were found and each $n$-layer state remains metastable up to $\eta_{\mathrm{p}}^{\mathrm{r}}=1.5$, a value close to the liquid-gas-crystal triple point according to free volume theory for the AO model [16]. Moreover, for large values of $\eta_{\mathrm{p}}^{\mathrm{r}}$ the numerical routine converges very rapidly, which hints at deep (nevertheless metastable) free energy minima for these layered surface states. 
As the layering transitions are thermodynamic surface phase transitions, they manifest themselves as discontinuous jumps in the Gibbs adsorption [30, 31],

$$
\Gamma_{i}=\int_{0}^{\infty} \mathrm{d} z\left(\rho_{i}(z)-\rho_{i}(\infty)\right),
$$

for both components $i=\mathrm{c}, \mathrm{p}$, and these can be obtained from equation (5) and

$$
\Gamma_{\mathrm{c}}=-\left.\frac{\partial \gamma_{\mathrm{wf}}}{\partial \mu_{\mathrm{c}}}\right|_{\mu_{\mathrm{p}}}, \quad \Gamma_{\mathrm{p}}=-\left.\frac{\partial \gamma_{\mathrm{wf}}}{\partial \mu_{\mathrm{p}}}\right|_{\mu_{\mathrm{c}}},
$$

where $\gamma_{\mathrm{wf}}$ is the wall tension of the fluid. Moving along the gas branch of the liquid-gas bulk binodal ties together changes in both chemical potentials:

$$
\left.\frac{\mathrm{d} \gamma_{\mathrm{wg}}}{\mathrm{d} \mu_{\mathrm{p}}}\right|_{\text {coex }}=\left.\frac{\partial \gamma_{\mathrm{wg}}}{\partial \mu_{\mathrm{p}}}\right|_{\mu_{\mathrm{c}}}+\left.\left.\frac{\partial \gamma_{\mathrm{wg}}}{\partial \mu_{\mathrm{c}}}\right|_{\mu_{\mathrm{p}}} \frac{\mathrm{d} \mu_{\mathrm{c}}}{\mathrm{d} \mu_{\mathrm{p}}}\right|_{\text {coex }},
$$

where the slope of the bulk binodal fulfils a Clapeyron-type equation,

$$
\left.\frac{\mathrm{d} \mu_{\mathrm{c}}}{\mathrm{d} \mu_{\mathrm{p}}}\right|_{\text {coex }}=-\frac{\Delta \rho_{\mathrm{p}}}{\Delta \rho_{\mathrm{c}}},
$$

which can be deduced from the Gibbs-Duhem equation in a straightforward fashion [43]. Here, $\Delta \rho_{i}=\rho_{i}^{1}-\rho_{i}^{\mathrm{g}}$ is the difference between the densities of species $i=\mathrm{c}, \mathrm{p}$ in the liquid and gas phases (note that $\Delta \rho_{\mathrm{p}}<0$ ). Hence, we obtain

$$
\left.\frac{\mathrm{d} \gamma_{\mathrm{wg}}}{\mathrm{d} \mu_{\mathrm{p}}}\right|_{\text {coex }}=-\Gamma_{\mathrm{p}}+\Gamma_{\mathrm{c}} \frac{\Delta \rho_{\mathrm{p}}}{\Delta \rho_{\mathrm{c}}},
$$

where the adsorptions $\Gamma_{\mathrm{c}}$ and $\Gamma_{\mathrm{p}}$ refer to those of the gas at bulk coexistence. Hence, crossing a layering transition (again at bulk coexistence) is necessarily accompanied by a jump in the adsorptions $\Gamma_{i}$; consequently via equation (10) this leads to a discontinuity of slope of the wall-gas interface tension. From Young's equation (1), it follows that this also leads to a jump in the slope of the contact angle, $\mathrm{d} \cos \theta / \mathrm{d} \eta_{\mathrm{p}}^{\mathrm{r}}$. This is consistent with our findings above of crossing of different branches, $\theta_{n-1}$ and $\theta_{n} .^{5}$

Note that all quantities in equation (10) can be independently obtained from our DFT results, namely the adsorption $\Gamma_{i}$ from the integral over the respective density profile, equation (6), the differences $\Delta \rho_{i}$ from the bulk phase diagram and the left-hand side of equation (10) from a numerical derivative of the results for $\gamma_{\mathrm{wg}}$, as obtained through equation (4). As a check for internal consistency of our calculations we have chosen the state point with $\eta_{\mathrm{p}}^{\mathrm{r}}=1.1$ for $q=1$ at bulk coexistence, which is very close to the binodal of the first layering transition (which is at $\eta_{\mathrm{p}}^{\mathrm{r}} \approx 1.104$, as discussed below). We find equation (10) to be fulfilled to three significant digits; for the zero-layer (one-layer) state both sides evaluate to $1.264 / \sigma_{\mathrm{c}}^{2}\left(1.352 / \sigma_{\mathrm{c}}^{2}\right)$. Together, these two estimates yield a jump in $\mathrm{d} \cos \theta / \mathrm{d} \eta_{\mathrm{p}}^{\mathrm{r}}$ of 0.247 , which is consistent with our data for the contact angle for $q=1$ (which gives 0.238 ) and which is discussed below.

We will now consider the case of larger polymer-colloid size ratios, $q=1$; see figure 5 for more detail. The zero-layer branch is calculated for a number of fugacities from high values, $\eta_{\mathrm{p}}^{\mathrm{r}}=3$, to the spinodal point close to $\eta_{\mathrm{p}}^{\mathrm{r}}=1$. The higher- $n$ branches are only calculated between 0.95 and $1.5(n=1)$ and between the wetting transition [31] and the crossing point of the zero-layer and the one-layer branch $(n=2-6$, which practically fall on top of each other). It is found that for this size ratio the zero-layer branch is stable everywhere except

\footnotetext{
5 The reported jump in $\gamma_{\mathrm{wg}}$ as a function of the difference of colloid packing fractions of the liquid and gas phases at the first layering transition [32] arises from crossing the adsorption spinodal rather than the binodal. The equilibrium curve for $\gamma_{\mathrm{wg}}$ is continuous.
} 


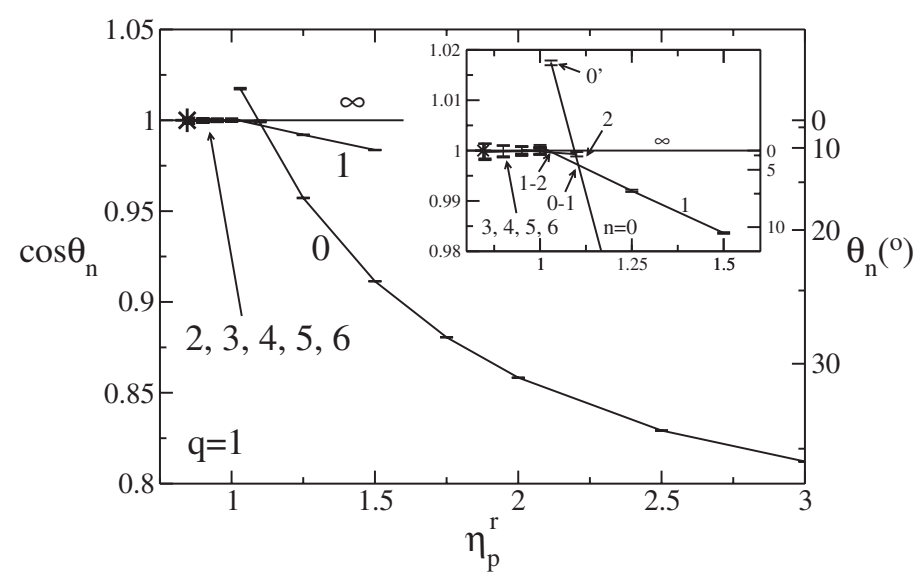

Figure 5. As figure 3, but for $q=1$. The inset displays a magnification of the area close to the wetting transition. The points marked $0-1$ and 1-2 are the first layering and second layering transitions, respectively, and the spinodal point of the zero-layer branch is denoted as $0^{\prime}$.

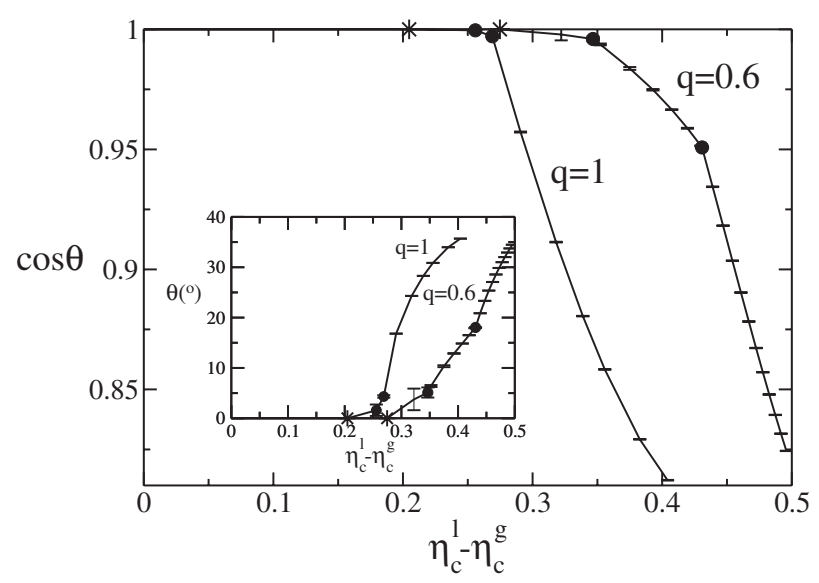

Figure 6. The equilibrium contact angle, $\cos \theta$ (main figure) and $\theta$ (in degrees, displayed in the inset), as a function of the difference in colloid packing fractions, $\eta_{\mathrm{c}}^{\mathrm{l}}-\eta_{\mathrm{c}}^{\mathrm{g}}$, of the coexisting bulk liquid and gas phases for two size ratios, $q=0.6$ and 1 . The locations of the equilibrium layering transitions are marked by filled circles. The stars mark the transitions from partial to complete wetting according to Brader et al [30, 31].

in a small regime of $\eta_{\mathrm{p}}^{\mathrm{r}}$ between 0.85 and 1.1 (where $\cos \theta_{n}$ is close to 1 ) and where layering transitions are located. We have been able to identify the locations of the first and second layering transitions; higher transitions are eroded by the numerical noise.

Figure 6 shows the resulting equilibrium values of $\cos \theta$ (see the inset for the bare angle $\theta$ ) as a function of the difference in colloid packing fractions of the coexisting liquid and gas phases, $\eta_{\mathrm{c}}^{\mathrm{l}}-\eta_{\mathrm{c}}^{\mathrm{g}}$, for both size ratios considered, $q=0.6$ and 1 . This representation enables one to make direct contact with experiments, where density differences of coexisting phases are rather directly accessible [7,9-11]. For both size ratios the contact angle is of the same order of magnitude, i.e. $\cos \theta=0.8-1$, but for $q=1$ the wetting transition lies closer (in this representation) to the bulk critical point. Only the first layering transition for $q=0.6$ occurs at a considerably larger contact angle of $\cos \theta_{0-1} \approx 0.95$ or $\theta_{0-1} \approx 18^{\circ}$. A remarkable fact is that 
for both size ratios, there is a substantial region in the partial wetting regime close to the wetting transition where the contact angle remains very small, i.e. the range $\left(\eta_{\mathrm{c}}^{1}-\eta_{\mathrm{c}}^{\mathrm{g}}\right) \approx 0.28-0.35$ for $q=0.6$ and $\left(\eta_{\mathrm{c}}^{\mathrm{l}}-\eta_{\mathrm{c}}^{\mathrm{g}}\right) \approx 0.2-0.27$ for $q=1$.

\subsection{Surface phase behaviour}

Layering transitions of the AO model at a hard wall were reported earlier by Brader et al [30,31] as obtained within the same DFT approximation [28, 29] and using numerical routines similar to those employed in the present work. However, in contrast to the present study, that of Brader et al determined (implicitly) spinodal points along two paths: first, by reducing $\eta_{\mathrm{p}}^{\mathrm{r}}$ at bulk (liquid-gas) coexistence and following the evolution of the zero-layer state, observing jumps to one-layer and subsequently higher-layer states, and second, by keeping $\eta_{\mathrm{p}}^{\mathrm{r}}$ fixed and approaching the gas branch of the binodal by increasing $\eta_{\mathrm{c}}$, starting from very small values. Results along reversed paths for the second case, i.e. decreasing $\mu_{\mathrm{c}}$ at constant $\eta_{\mathrm{p}}^{\mathrm{r}}$, were taken to ascertain that hysteresis effects are small, and it was concluded that the spinodal points give reasonable indications of the locations of the equilibrium layering transitions [31, 44]. We will discuss in the following the relation of these findings to those of the present work. For this purpose, we have chosen a reference case, i.e. the first layering transition for $q=1$, and mapped out its surface phase behaviour completely, i.e. including binodal and spinodal lines off bulk coexistence. The result is plotted in figure 7 .

The layering transition binodal is obtained by determining, for each value of $\eta_{\mathrm{p}}^{\mathrm{r}}$, the value of $\eta_{\mathrm{c}}$ at which the zero-layer and one-layer states have equal interface tensions and are thus in thermodynamic coexistence. The resulting binodal line extends below the first layering transition at bulk coexistence, at $\eta_{\mathrm{p}, 0-1}^{\mathrm{r}} \approx 1.1$, to lower values of $\eta_{\mathrm{p}}^{\mathrm{r}}$ into the bulk gas phase region. As this is a transition between two (surface) phases of the same symmetry, a van der Waals loop in the free energy and a critical point are mandatory. In order to find the location of the critical point, we fit our data for the colloid adsorption $\Gamma_{\mathrm{c}}\left(\eta_{\mathrm{p}}^{\mathrm{r}}\right)$ of the coexisting zero-layer and one-layer states with a fourth-order polynomial, $\eta_{\mathrm{p}}^{\mathrm{r}}=a_{0}+a_{2}\left(\Gamma_{\mathrm{c}}-a_{1}\right)^{2}+a_{3}\left(\Gamma_{\mathrm{c}}-a_{1}\right)^{3}+a_{4}\left(\Gamma_{\mathrm{c}}-a_{1}\right)^{4}$, where the $a_{i}$ are free fitting parameters; see figure 7. The value of $a_{0}$ is an estimate of the critical value of $\eta_{\mathrm{p}}^{\mathrm{r}}$, and the functional form is chosen to yield the mean-field critical exponent of $1 / 2$, i.e. $\left(\Gamma_{\mathrm{c}}-a_{1}\right) \sim\left(\eta_{\mathrm{p}}^{\mathrm{r}}-a_{0}\right)^{1 / 2}$, as the DFT is a mean-field theory in the sense that it does not capture fluctuation effects. The accuracy of the colloid packing fraction $\eta_{\mathrm{c}}$ of points on the layering binodal is high; errors are typically smaller than $0.1 \%$ of $\eta_{\mathrm{c}}$. However, the critical point is an extrapolation and is therefore much more sensitive to errors, i.e. these may be as large as $3 \%$ in $\eta_{\mathrm{p}}^{\mathrm{r}}$ and $1 \%$ in $\eta_{\mathrm{c}}$. We have also located the zero-layer spinodal by taking paths at constant $\eta_{\mathrm{p}}^{\mathrm{r}}$ and increasing values of $\eta_{\mathrm{c}}$, monitoring the stability of the solution under the iteration procedure. The values of $\eta_{\mathrm{c}}$ where the zero-layer state becomes unstable and converges to the one-layer state define the 'adsorption spinodal', located at larger values at $\eta_{\mathrm{c}}$ compared to the layering binodal. (The precise location of spinodal points is governed by the numerical resolution of the step size in $\eta_{\mathrm{c}}$, i.e. typically $1 \%$ in $\eta_{\mathrm{c}}$.) Similarly, we have investigated the stability of the one-layer states upon reducing $\eta_{\mathrm{c}}$ at constant $\eta_{\mathrm{p}}^{\mathrm{r}}$. This defines the 'desorption spinodal', where the onelayer solutions converge to the zero-layer states and which is located at smaller values of $\eta_{\mathrm{c}}$ as compared to the layering binodal. Upon decreasing $\eta_{\mathrm{p}}^{\mathrm{r}}$, both spinodals and the layering binodal end at the surface critical point. Indeed even for values of $\eta_{\mathrm{p}}^{\mathrm{r}}$ quite far above the layering critical point the adsorption and desorption spinodals are very close and we can confirm the finding of Brader et al that hysteresis effects at constant $\eta_{\mathrm{p}}^{\mathrm{r}}$ are small.

Next, we discuss the various aspects of this surface phase transition in relation to bulk liquid-gas coexistence. We first note that no intrinsic difference is observed between the 


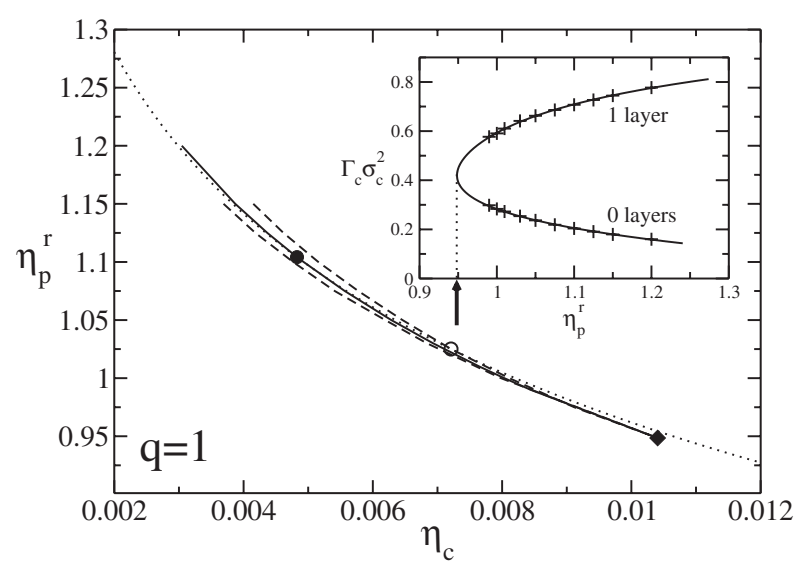

Figure 7. The full surface phase diagram of the first layering transition (from the zero-layer to the one-layer state) for $q=1$ as a function of (bulk) colloid packing fraction, $\eta_{\mathrm{c}}$, and polymer reservoir packing fraction, $\eta_{\mathrm{p}}^{\mathrm{r}}$. Shown is the layering binodal (full curve), the one-layer desorption spinodal (dashed curve, left), the zero-layer adsorption spinodal (dashed curve, right) and the gas branch of the bulk liquid-gas binodal (dotted curve). The point where the layering binodal crosses the bulk liquid-gas binodal is the first layering transition at bulk coexistence (filled circle). The point where the zero-layer adsorption spinodal line hits the bulk binodal is the zero-layer spinodal point at bulk coexistence (open circle), according to Brader et al [31]. Both spinodals and the binodal terminate at the layering critical point (filled diamond) located off bulk coexistence in the one-phase gas region. Note that the one-layer desorption spinodal runs entirely in the one-phase gas region and hence does not cross the bulk binodal. The inset shows the colloid adsorption, $\Gamma_{\mathrm{c}} \sigma_{\mathrm{c}}^{2}$, of the coexisting zero-layer and one-layer states (along the layering binodal) as a function of the polymer reservoir packing fraction, $\eta_{\mathrm{p}}^{\mathrm{r}}$; the dividing surface is chosen at $z=\sigma_{\mathrm{c}} / 2$ (the lower bound of the integral in equation (6)). The symbols are obtained from DFT, the full curve is the fit (see the text) and the arrow at the horizontal axis denotes the estimated value of $\eta_{\mathrm{p}}^{\mathrm{r}}$ at the layering critical point.

layering phase transition in the stable gas region and that in the two-phase region where the gas is metastable. In the following, we consider the three different surface phase transition lines, i.e. the adsorption spinodal, layering binodal and desorption spinodal, and their relation to the bulk binodal. First, the crossing point of the adsorption spinodal and the bulk liquid-gas binodal denotes the spinodal point terminating the metastability region of the zero-layer state upon reducing $\eta_{\mathrm{p}}^{\mathrm{r}}$ at bulk coexistence. This corresponds to the adsorption spinodal point of the contact angle (see figure 5), as located previously by Brader et al (as well as other adsorption spinodal points at bulk coexistence for different size ratios, $q=0.6,0.7,1)$. Our numerical value of $\eta_{\mathrm{p}}^{\mathrm{r}}$ agrees well with that of [31]. Second, the point of crossing between the layering binodal and bulk binodal is the layering transition at bulk coexistence (accompanied by a kink in the contact angle, as outlined above). This state point can be seen as a triple point between the bulk liquid and two different surface states of the bulk gas. The location of this triple point is quite different from the adsorption spinodal point at bulk coexistence. Although hysteresis for a path at constant $\eta_{\mathrm{p}}^{\mathrm{r}}$ is small, this is not the case for the path along bulk coexistence, due to the fact that the gas branch of the bulk binodal and the layering (spinodal and binodal) lines have very similar slopes. Hence the location of any crossing point is very sensitive to the precise location of the individual lines. Third, in striking contrast to the previous two cases, the desorption spinodal does not cross the bulk binodal, but remains in the one-phase gas region for increasing values of $\eta_{\mathrm{p}}^{\mathrm{r}}$. We have checked this for one additional path at $\eta_{\mathrm{p}}^{\mathrm{r}}=2$, starting with a one-layer profile at bulk coexistence and decreasing $\eta_{\mathrm{c}}$ and indeed found the 


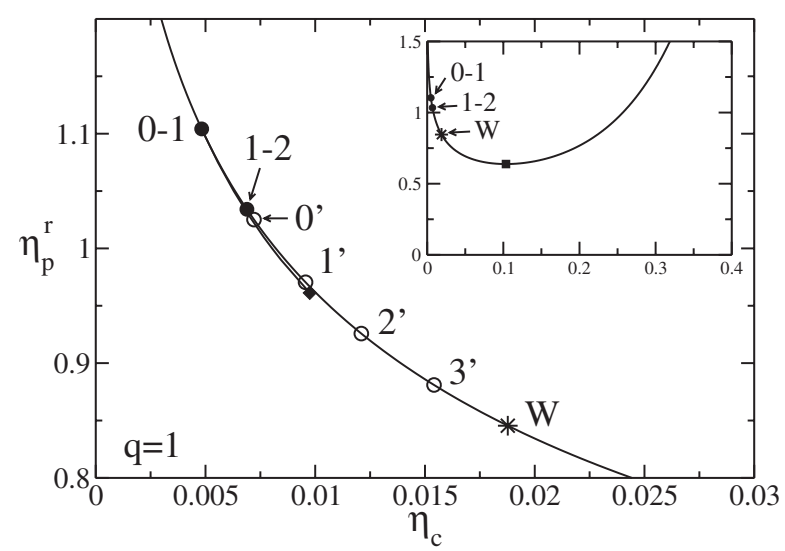

Figure 8. A summary of the known features of the surface phase diagram of the AO model at a hard wall for size ratio $q=1$ as a function of the colloid packing fraction, $\eta_{\mathrm{c}}$, and polymer reservoir packing fraction, $\eta_{\mathrm{p}}^{\mathrm{r}}$. Shown are the gas branch of the bulk binodal (full curve) and equilibrium first (filled circle, marked 0-1) and second (filled circle, marked 1-2) layering transitions at bulk coexistence, as obtained from the present work. The first layering critical point (diamond) is connected via the layering binodal line (full curve) to the first layering transition at bulk coexistence. The spinodal lines are omitted for clarity. The zero-, one-, two-, three-layer adsorption spinodal points at bulk coexistence (open circles, marked $0^{\prime}, 1^{\prime}, 2^{\prime}$ and $3^{\prime}$ respectively) and the wetting transition (star, marked W) are taken from [31]. The inset shows some of the data together with the bulk critical point (large filled square) on a larger scale.

desorption spinodal point in the one-phase gas region. This behaviour is consistent with the behaviour of the contact angle, which we discussed above for $q=0.6$ and 1 , and where adsorption spinodal points were found upon decreasing $\eta_{\mathrm{p}}^{\mathrm{r}}$, but no desorption spinodal points were found upon increasing $\eta_{\mathrm{p}}^{\mathrm{r}}$. Although we have determined this scenario only extensively for the first layering transition for $q=1$, we believe it to hold more generally for higher layering transitions and other size ratios.

In order to summarize our present results and those of [31] for the layering transitions of the AO model at a hard wall, we draw the surface phase diagram for $q=1$ in figure 8 . The results for the $n$-layer adsorption spinodal points for $n=0,1,2,3$ at bulk coexistence are taken from [31]. At bulk coexistence, the first and the second layering binodal points are located at higher fugacities compared to the corresponding zero-layer and one-layer adsorption spinodal points, respectively. Higher layering transitions, corresponding to two- and threelayer spinodal points of Brader et al do not emerge from our present data. On the other hand, Brader et al have not found layering lines extending into the bulk gas phase for $q=1$, which we have located for the first layering transition. We have not searched for a similar layering line in the case of the second layering transition. However, from the topology of the first layering transition, which we established above, and taking into account the considerable separation of the second layering binodal and the one-layer adsorption spinodal points at bulk coexistence, it seems plausible that such a layering binodal line also exists for the second layering transition.

Next, we compare our results to those from simulations by Dijkstra and van Roij for $q=1$ [21]. They find different regimes of complete and partial wetting, and first, second and third layering transitions located off bulk coexistence. Our results for the first layering binodal line extending into the one-phase gas region are in qualitative agreement with their findings. Previous comparisons with DFT results [31] left a puzzle, because a layering line was found for $q=0.6$, but not for $q=1$. The length of this first layering binodal line obtained from 


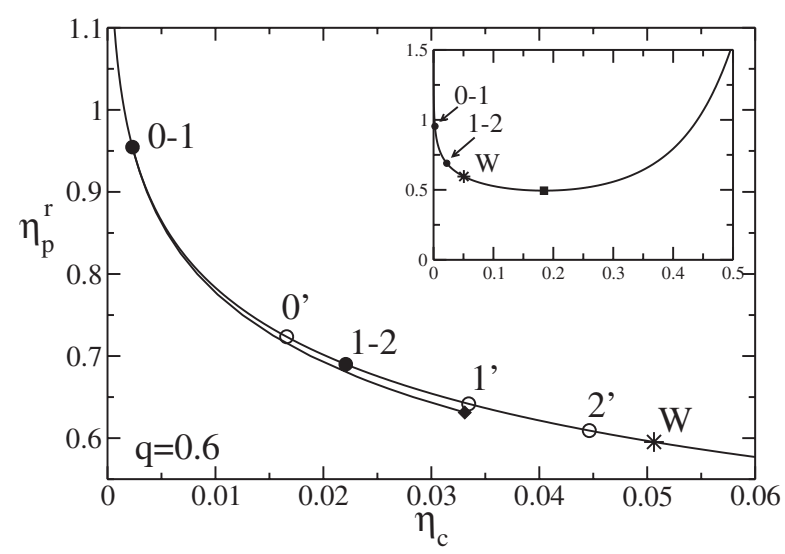

Figure 9. As figure 8, but for $q=0.6$. The zero-layer adsorption spinodal $[30,31]$ connecting state point $0^{\prime}$ with the surface critical point (diamond) is omitted for clarity.

DFT is considerably larger (in the range of $\eta_{\mathrm{p}}^{\mathrm{r}}$ ) than that found in simulations [21]. With hindsight, this deviation seems consistent with that in other situations, as too small values for the critical polymer fugacities compared to simulations are also found in bulk (see e.g. [23]) or in confinement in planar capillaries $[45,46]$. We have not obtained results for the higher- $n$ layering binodal lines, so we cannot compare the DFT for $n>1$ efficiently with simulations. Nevertheless, the overall agreement is good and the DFT seems to capture all relevant effects: i.e. the layering transitions at bulk coexistence, a layering line extending into the one-phase region and a wetting transition [31]. Given the approximate nature of the free energy functional and the fact that the surface phase transitions are governed by tiny free energy differences, this is quite remarkable.

Coming back to $q=0.6$, we summarize our results and that of Brader et al [30,31] in figure 9. Similar to the case for $q=1$, the first and second layering transitions at bulk coexistence are located at higher fugacities than the corresponding zero-layer and one-layer adsorption spinodal points, respectively. The effect, however, is more dramatic than for $q=1$; see figure 8. Brader et al have also found a two-layer adsorption spinodal point, indicating the existence of a third layering transition, whose binodal we have not been able to locate. We have also determined the first layering line extending into the bulk gas phase and find it to be remarkably long. The location of the critical point is determined with the same fitting procedure as described above for the first layering transition for $q=1$. The large separation along the bulk binodal of the second layering transition and the one-layer adsorption spinodal point suggests again the presence of a second layering line.

\section{Conclusion}

In conclusion, we have investigated the contact angle, $\theta$, of the (colloidal) liquid-gas interface and a hard wall using the AO model colloid-polymer mixture and considering two different polymer-to-colloid size ratios, $q=0.6$ and 1 . Our results for $\theta$ are obtained via Young's equation from independent numerical DFT calculations of the liquid-gas, the wall-gas and the wall-liquid interfacial free energies at bulk coexistence. At the planar wall-gas interface at bulk coexistence, we identify a range of different metastable states each corresponding to a number of adsorbed colloid layers at the wall. We argue that the globally stable state 
corresponds to the lowest wall-gas interface tension, and therefore possesses the largest value of $\theta$. For small density differences of the coexisting liquid and gas, i.e. close to the bulk critical point, the wall is completely wetted by colloidal liquid $[30,31]$ and, hence, $\theta=0$. Moving along bulk coexistence, away from the critical point, the wetting transition [30, 31] is crossed, and $\theta$ becomes non-zero. However, typical values of $\theta$ remain very small over a considerable range of state points in the partial wetting regime. Mediated by a sequence of first-order layering transitions at the wall-gas interface, the contact angle grows upon moving further from the critical point and reaches typical values up to $\sim 35^{\circ}$. These layering transitions of the coexisting colloidal gas in contact with the wall appear as discontinuities in the slope of $\theta$ as a function of a thermodynamic control parameter, e.g. the polymer reservoir packing fraction or the colloid packing fraction difference between the two coexisting bulk phases.

Previous difficulties in measuring the contact angle accurately [7] have been overcome by Aarts and Lekkerkerker with the use of confocal scanning laser microscopy [9] in a system with $q=0.56$. The authors conclude that $\theta=0$ for all state points considered, consistent with their direct observation of a prominent colloid wetting film at the interface of the bulk gas with the wall. They point out that actual values of $\theta$ are very sensitive to the precise determination of the location of the wall. Large values of contact angles as well as the observation of the transition to complete wetting have been reported by Wijting et al $[10,11]$, using extrapolation of dynamical measurements (i.e. moving the wall) to zero velocity. However, some reservations have been voiced with respect to the latter results [9]. The magnitude of the contact angle results from subtle differences between the interface tensions and we do not expect our present results to resolve experimental issues. The contact angles which we have calculated for the highly idealized AO model in contact with a hard wall can therefore only serve as a reference case. Important effects due to more realistic polymer-polymer interactions [34], polydispersity and gravity are not captured in our present model (see [31] for a discussion).

We have also reconsidered the surface phase behaviour of the AO model colloid-polymer mixture at a hard wall. This system is known to exhibit a sequence of first-order layering transitions upon following the gas branch of the liquid-gas bulk binodal towards the bulk critical point (i.e. reducing $\eta_{\mathrm{p}}^{\mathrm{r}}$ ). In addition, layering lines extending off bulk coexistence into the one-phase gas region have been located. Such a layering transition is characterized by a jump in the colloid adsorption at the wall and can be identified as the growth of an additional colloid layer at the wall-gas interface [21, 30, 31]. For one specific case, the first layering transition for size ratio $q=1$, we have determined the layering binodal, which gives the equilibrium location of the transition, to high accuracy. In addition, we have located the zero-layer adsorption spinodal line, beyond which (for higher $\eta_{\mathrm{c}}$ at constant $\eta_{\mathrm{p}}^{\mathrm{r}}$ ) the zero-layer state is unstable and the one-layer desorption spinodal line, marking the end of stability of the one-layer state (i.e. for lower $\eta_{\mathrm{c}}$ at constant $\eta_{\mathrm{p}}^{\mathrm{r}}$ ). The layering binodal and the adsorption and desorption spinodal lines end at a critical point, located in the single-phase gas region of the bulk phase diagram. The crossing point of the layering binodal and the bulk binodal represents a triple point between the bulk liquid and the two layered states ( 0 and 1 layers) of the bulk gas which have different values of adsorption of the two components. We find the location of this triple point to differ substantially from the (previously identified [30, 31]) crossing point of the adsorption spinodal and the bulk binodal. Remarkably, we could not find a crossing point of the desorption spinodal and the bulk binodal and its absence gives rise to continued (meta)stability of the one-layer state upon increasing $\eta_{\mathrm{p}}^{\mathrm{r}}$ at coexistence. We believe that this scenario holds for higher layering transitions and other size ratios. We have presented further results for the second layering transition for $q=1$ as well as for first and second layering transitions for $q=0.6$. The order of the wetting transition and whether it occurs via an infinite or a finite sequence of layering transitions remain open questions. Whether 
the occurrence of layering transitions is specific to the AO model (see also [31] for a more extensive discussion) or would be present in more realistic descriptions of colloid-polymer mixtures is another interesting question. However, any experimental attempt to reveal such (layering) phase behaviour would require exceptional accuracy for determining $\theta$ or resolution on the particle level for direct observation.

\section{Acknowledgments}

D G A L Aarts is thanked for pointing out the relevance of the wall contact angle of the colloidal liquid-gas interface to us. We acknowledge useful discussions and correspondence with R Evans, J M Brader, R Roth and M Dijkstra. R Evans is also thanked for critically reading what turned out to be a preliminary version of our manuscript and $\mathrm{R}$ Roth for an independent check of numerical results. This work is financially supported by the SFB-TR6 programme 'Physics of colloidal dispersions in external fields' of the Deutsche Forschungsgemeinschaft (DFG). The work of MS is part of the research programme of the Stichting voor Fundamenteel Onderzoek der Materie (FOM), which is financially supported by the Nederlandse Organisatie voor Wetenschappelijk Onderzoek (NWO).

\section{References}

[1] Poon W C K 2002 J. Phys.: Condens. Matter 14 R859

Tuinier R, Rieger J and de Kruif C G 2003 Adv. Colloid Interface Sci. 1031

[2] Löwen H 2001 J. Phys.: Condens. Matter 13 R415

[3] de Hoog E H A and Lekkerkerker H N W 1999 J. Phys. Chem. B 1035274

[4] de Hoog E H A, Lekkerkerker H N W, Schulz J and Findenegg G H 1999 J. Phys. Chem. B 10310657

[5] Chen B-H, Payandeh B and Robert M 2000 Phys. Rev. E 622369

[6] Chen B-H, Payandeh B and Robert M 2001 Phys. Rev. E 64042401

[7] Aarts D G A L, van der Wiel J H and Lekkerkerker H N W 2003 J. Phys.: Condens. Matter 15 S245

[8] Aarts D G A L, Schmidt M and Lekkerkerker H N W 2004 Science 304847

[9] Aarts D G A L and Lekkerkerker H N W 2004 Proc. CODEF Conf.; J. Phys.: Condens. Matter 16 S4231

[10] Wijting W K, Besseling N A M and Cohen Stuart M A 2003 Phys. Rev. Lett. 90196101

[11] Wijting W K, Besseling N A M and Cohen Stuart M A 2003 J. Phys. Chem. B 10710565

[12] Asakura S and Oosawa F 1954 J. Chem. Phys. 221255

[13] Asakura S and Oosawa F 1958 J. Polym. Sci. 33183

[14] Vrij A 1976 Pure Appl. Chem. 48471

[15] Gast A P, Hall C K and Russell W B 1983 J. Colloid Interface Sci. 96251

[16] Lekkerkerker H N W, Poon W C K, Pusey P N, Stroobants A and Warren P B 1992 Europhys. Lett. 20559

[17] Meijer E J and Frenkel D 1991 Phys. Rev. Lett. 671110

[18] Meijer E J and Frenkel D 1994 J. Chem. Phys. 1006873

[19] Dijkstra M, Brader J M and Evans R 1999 J. Phys.: Condens. Matter 1110079

[20] Bolhuis P G, Louis A A and Hansen J-P 2002 Phys. Rev. Lett. 89128302

[21] Dijkstra M and van Roij R 2002 Phys. Rev. Lett. 89208303

[22] Vink R L C and Horbach J 2004 Preprint cond-mat/0310404

[23] Vink R L C and Horbach J 2004 Proc. CODEF Conf.; J. Phys.: Condens. Matter 16 S3807

[24] Dzubiella J, Jusufi A, Likos C N, von Ferber C, Löwen H, Stellbrink J, Allgaier J, Richter D, Schofeld A B, Smith P A, Poon W C K and Pusey P N 2001 Phys. Rev. E 64010401

[25] Vrij A 1997 Physica A 235120

[26] Brader J M and Evans R 2000 Europhys. Lett. 49678

[27] Brader J M, Dijkstra M and Evans R 2001 Phys. Rev. E 63041405

[28] Schmidt M, Löwen H, Brader J M and Evans R 2000 Phys. Rev. Lett. 851934

[29] Schmidt M, Löwen H, Brader J M and Evans R 2002 J. Phys.: Condens. Matter 149353

[30] Brader J M, Evans R, Schmidt M and Löwen H 2002 J. Phys.: Condens. Matter 14 L1

[31] Brader J M, Evans R and Schmidt M 2003 Mol. Phys. 1013349

[32] Wessels P P F, Schmidt M and Löwen H 2004 J. Phys.: Condens. Matter 16 L1 
[33] Fortini A et al 2004 in preparation

[34] Aarts D G A L, Dullens R P A, Lekkerkerker H N W, Bonn D and van Roij R 2004 J. Chem. Phys. 1201973

[35] Rowlinson J S and Widom B 2002 Molecular Theory of Capillarity 1st edn (New York: Dover)

[36] Sullivan D E and Telo da Gama M M 1986 Wetting transitions and multilayer adsorption at fluid interfaces Fluid Interfacial Phenomena ed C A Croxton (New York: Wiley) p 45

[37] Dietrich S 1988 Wetting phenomena Phase Transitions and Critical Phenomena vol 12, ed C Domb and J L Lebowitz (London: Academic) p 1

[38] Evans R 1992 Fundamentals of Inhomogeneous Fluids ed D Henderson (New York: Dekker) chapter 3 (Density Functionals in the Theory of Nonuniform Fluids) p 85

[39] Broyles A A 1960 J. Chem. Phys. 33456

[40] Evans R, Henderson J R, Hoyle D C, Parry A O and Sabeur Z A 1993 Mol. Phys. 80755

[41] Evans R, Leote de Carvalho R J F, Henderson J R and Hoyle D C 1994 J. Chem. Phys. 100591

[42] Henderson J R 1994 Phys. Rev. E 504836

[43] Schmidt M, Dijkstra M and Hansen J P 2004 unpublished

[44] Brader J M and Evans R 2004 private communication

[45] Schmidt M, Fortini A and Dijkstra M 2003 J. Phys.: Condens. Matter 48 S3411

[46] Schmidt M, Dijkstra M and Hansen J P 2004 Proc. CODEF Conf.; J. Phys.: Condens. Matter 16 S4185 